\title{
All-Optical Polarization Control Through Brillouin Amplification
}

\author{
Luc Thévenaz, Avi Zadok* ${ }^{\dagger}$, Avishay Eyal* and Moshe Tur* \\ Ecole Polytechnique Fédérale de Lausanne, STI-NAM Station 11, CH-1015 Lausanne, Switzerland \\ * School of Electrical Engineering, Faculty of Engineering, Tel-Aviv University, Tel-Aviv 69978, Israel \\ + Presently with the Department of Applied Physics, MC 128-95, California Institute of Technology, Pasadena, CA 91125, USA \\ luc.thevenaz@epfl.ch,.avizadok@,caltech.edu,avishay@eng.tau.ac.il,tur@eng.tau.ac.il
}

\begin{abstract}
Amplification through stimulated Brillouin scattering in optical fibers can totally modify the polarization properties of the light, enabling an all-optical polarization control and a direct estimate on the fiber birefringence.
\end{abstract}

(C)2008 Optical Society of America

OCIS codes: (290.5900) Scattering, Stimulated Brillouin; (120.5410) Polarimetry.

\section{Introduction}

Stimulated Brillouin Scattering (SBS) has the lowest threshold power of all the nonlinear effects observed in silica optical fibers. SBS has found a broad range of applications, such as distributed fiber sensing [1, 2], fiber laser [3], and generation of slow \& fast light $[4,5]$. SBS can be regarded as a parametric process, involving two optical waves (pump and signal) and an idler acoustic wave. The beating of the two optical fields enhances the acoustic wave through electrostriction, and the acoustic wave in turn generates a traveling grating in the fiber refractive index due to the elasto-optic effect [6]. The index grating can effectively couple the two optical waves, provided that strict phase-matching conditions are maintained [6]. These conditions imply that the optical waves must be counterpropagating, and require a specific value $\Omega_{B} / 2 \pi$ for the difference between their optical frequencies. This difference $\Omega_{B} / 2 \pi$ lies between $10-11 \mathrm{GHz}$ in silica fibers at $1550 \mathrm{~nm}$, and it is mainly determined by the acoustic velocity in silica. The tolerance on $\Omega_{B} / 2 \pi$ is very severe and results in an extremely narrowband process $(\sim 30 \mathrm{MHz})$. The net result of the interaction is a power transfer from the higher frequency optical wave to the lower frequency wave, giving rise to a gain process for the latter wave and a loss for the former. Since one of the driving mechanisms in SBS is the beating of two optical waves, the interaction is strongly and inherently polarizationdependent. For example, SBS vanishes for orthogonally polarized fields, and reaches maximum interaction for aligned polarizations. The polarization properties of this interaction have been so far poorly exploited, even though they are widely known and documented [7].

In this contribution we show that the polarization dependence of SBS offers very interesting tools for the investigation of polarization properties in optical fibers, and for the control of the state of polarization (SOP) of an optical wave. We experimentally show that the amplification through SBS in a medium with a weak random birefringence, such as a long standard fiber, results in a gain that varies between minimal and maximal values, according to the relation between the SOPs of the pump and the signal. Finally, we demonstrate a very promising and unique feature of Brillouin amplification: the possibility to radically transform the signal SOP, and to drag it so that it would match that of the pump. This last feature allows the implementation of a novel function, that of a polarization transformer in which the light exits with a definite and fixed SOP for any input random SOP.

\section{Polarization dependence of stimulated Brillouin scattering}

SBS is governed by a set of three coupled equations, describing the coupled evolution of the pump and signal optical waves and that of the acoustic wave [6]. Under the assumptions that the pump experiences negligible depletion, and that the optical waves only experience negligible changes along the typical damping distance of the acoustic wave ( $\sim 0.1 \mathrm{~mm}$ in silica), the set of three equations may be reduced to only one, describing the signal wave:

$$
d \vec{E}_{s}=\frac{1}{2} g(v)\left(\vec{E}_{s} \cdot \vec{E}_{p}^{*}\right) \vec{E}_{p} d z
$$




\section{OML7.pdf}

In Eq. (1), $\vec{E}_{p}$ and $\vec{E}_{s}$ are the pump and signal fields respectively and $g(v)$ is the linear Brillouin gain coefficient. The dot product $\left(\vec{E}_{s} \cdot \vec{E}_{p}^{*}\right)$ indicates that the local gain is polarisation-dependent, being maximal for aligned fields and zero for crossed fields. Since the optical waves are counterpropagating, aligned fields do not necessarily correspond to identical SOPs for pump and signal. Maximum interaction is achieved when the signal Jones vector is the complex conjugate of that of the pump [7]. This condition implies, for example, identical SOP's for linear polarizations, but opposite SOP's for circular polarisations.

This polarization dependence has important consequences for SBS. Let $\mathbf{d T} \mathbf{T}_{p}(z)$ and $\mathbf{d T} \mathbf{T}_{s}(z)$ denote the Jones matrices describing the SOP evolution of counterpropagating pump and signal waves, respectively, along an infinitesimal section of fiber at position $z$. In the absence of pump-signal interaction and for a low enough birefringence, $\mathbf{d T}_{p}(z)=\mathbf{d T}_{s}^{T}(z)$, and the evolution of the SOPs of the two optical waves as a function of position will be strongly correlated. For example, if the pump and the signal have linear and aligned polarizations at one point, they will be also aligned and linear at any other point where the pump SOP is linear. They will thus produce maximal interference and SBS gain at these positions. Continuing that scenario, if the pump has a circular SOP at some point, the signal field will rotate in the opposite direction at that position, the interferences will be null and the SBS gain zero. We refer to this scenario as the "aligned linear" case. Similarly, if pump and signal have linear and orthogonal SOPs at some point, they will be also linear and orthogonal at any other point where the pump SOP is linear, causing no interference and zero SBS gain. They will be also simultaneously circular, but with an identical sense of rotation for the fields causing maximal interaction. This scenario is referred to as the "orthogonal linear" case.

In a medium with random birefringence, the SOP of an optical wave also varies randomly during propagation. According to the Muller matrix description, the probability of having a linear polarization in a given location is twice larger than that of finding a circular polarization. This means that the aligned linear case will lead to the maximal gain, whereas the orthogonal linear situation will produce minimal, however non zero, SBS signal gain [7]. Theoretically the minimal gain $G \equiv \ln \left(P_{s}^{\text {out }} / P_{s}^{\text {in }}\right), P_{s}^{\text {out }} / P_{s}^{\text {in }}$ denoting input and output signal powers, would be half of the maximal one.

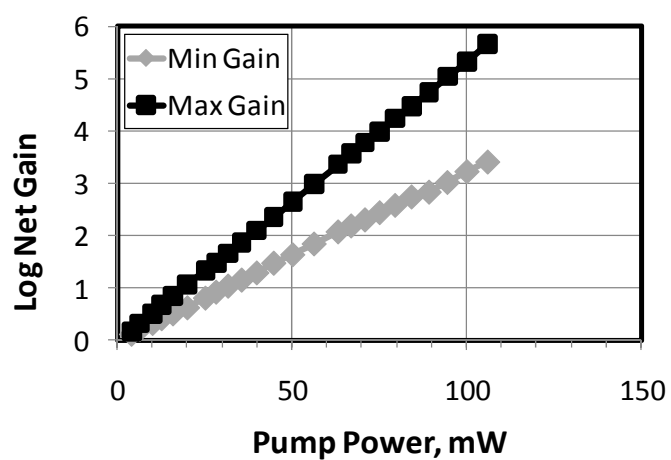

Fig. 1 Measured SBS gain as function of pump power in a $415 \mathrm{~m}$ SMF28 optical fiber. The signal input SOP was adjusted to give the maximum and minimum gain, respectively. All other input SOPs give intermediate gains.

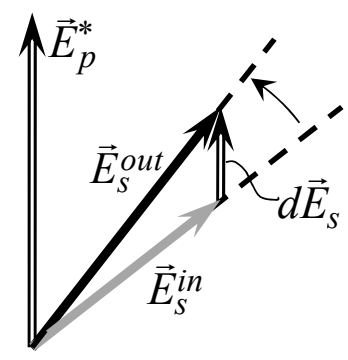

Fig. 2 Effect of incremental SBS gain on signal wave SOP. $d \vec{E}$ : fraction of the pump field transferred to the signal through SBS

For a given pump SOP, each of the two situations considered above corresponds to a specific signal SOP at the fiber input. These two input SOPs are orthogonal, and they would lead to maximum and minimum SBS signal gain. Any other setting will produce an intermediate gain between these maximal and minimal values. This dependence on input signal SOP was experimentally observed, and it is shown in Fig. 1. The linear gain $G$ was measured as a function of the pump power for the two settings of the signal SOP giving the maximal and the minimal gain. The ratio between these extreme gain values is not exactly 2 , probably because a perfect setting of the SOP is very difficult, and due to imperfect statistical mixture of the SOPs along the relatively short length of fiber.

Another consequence of Eq. 1 is that the increment $d \vec{E}_{s}$ of the signal field amplitude due to SBS is parallel to $\vec{E}_{p}$ from a field vector point of view. In other words the signal SOP is changed by the interaction, and the signal field increment is aligned with the pump SOP, as shown in Fig. 2. The effect of SBS amplification will be to gradually drag the signal field direction towards the pump field direction. For high amplification the dragging effect 
is so strong that the signal field would eventually align with the pump field. From that position onwards, the signal will experience maximal gain as described before.

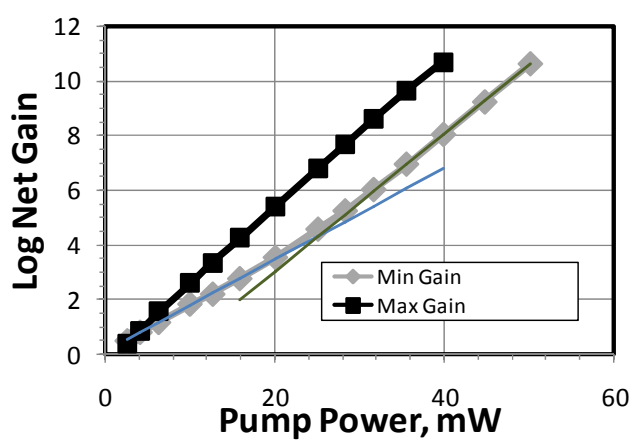

Fig.3 Measured SBS gain as function of pump power in a $2244 \mathrm{~m}$ dispersionshifted optical fiber. The signal input SOP was adjusted to give the maximum and minimum gain, respectively. The thin lines indicate the slopes for low and high gain regions in the minimum gain situation, showing at their intersection the transition (pump power $24 \mathrm{~mW}$ ) to full polarization pulling.

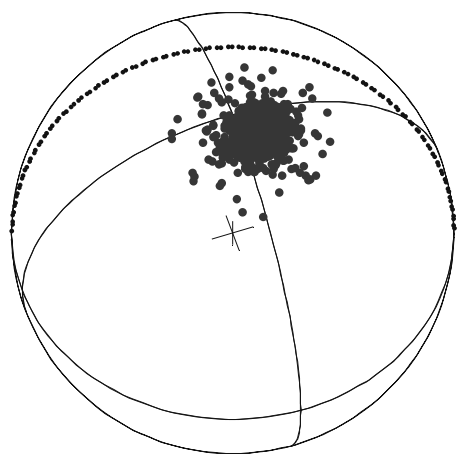

Fig.4 Output SOP measured at the end of a long optical fiber subject to SBS amplification with high gain $(\sim 35 \mathrm{~dB})$. The input SOP is fully scrambled, illustrating output SOP locking

This feature is illustrated in Fig. 3. Once a certain threshold pump power is reached, the slope of the minimum gain curve changes abruptly. Above this transition point, the slope is identical to that of the maximal gain curve. This change in the slope indicates that an effective alignment of the signal and pump fields had been reached. It must be pointed out that no slope change is observed for maximal gain, since pump and signal are already in the best alignment situation.

The position of the transition point in the minimum gain $v s$. pump power graph is determined by the competition between SBS gain and birefringence. Clearly its position is an indication of the amount of birefringence present in the fiber, as supported by measurements in segments of diverse fibers. Measurement of this transition point may provide a simple mean to evaluate the average birefringence of a fiber. A quantitative model for the interplay of SBS amplification and random birefringence is presently under study.

Finally, the polarization properties of SBS enable a novel, attractive all-optical technique for polarization control. By simply propagating the optical wave in a segment of fiber showing a modest birefringence and subject to a strong Brillouin amplification, the output SOP of polarization will always be quasi-equal to the complex conjugate of the pump SOP, for any signal input SOP. The degree of matching with the pump SOP depends on the Brillouin gain and the birefringence, but the signal output SOP can easily be kept within \pm 5 degrees of the pump SOP with gains larger than $30 \mathrm{~dB}$. This result is illustrated in Fig. 4, where the signal output SOP is measured while the input SOP is totally scrambled. This scheme can be a very valuable tool to fix the SOP before a coherent detection system, following propagation in a medium with random polarization properties such as a long fiber.

\section{Conclusions}

SBS has very unique polarization properties that have not been exploited so far. It can provide a simple tool to investigate birefringence properties of an optical fiber, and it enables simple all-optical locking of random input SOP to an arbitrary output SOP. A theoretical model supporting the experimental observation is being developed.

This work was realized in the framework of the European COST Action 299 "FIDES".

\section{References}

[1] T. Horiguchi, T. Kurashima, and M. Tateda, "A technique to measure distributed strain in optical fibers," IEEE Photon. Technol. Lett. Vol. 2, pp. 352-354, May 1990

[2] M. Nikles, L. Thevenaz, and P. Robert, "Brillouin gain spectrum characterization in single-mode optical fibers," J. of Lightwave Technol. Vol. 15, pp. 1842-1851, Oct. 1997

[3] J. C. Yong, L. Thévenaz, and B. Y. Kim, "Brillouin fiber laser pumped by a DFB laser diode," J. of Lightwave Technol. Vol. 12, pp. 546554, Feb. 2003

[4] M. González-Herráez, K.-Y. Song, and L. Thévenaz, "Optically controlled slow and fast light in optical fibers using stimulated Brillouin scattering,” Appl. Phys. Lett. Vol. 87, 081113, Aug. 2005.

[5] M. D. Stenner, M. A. Neifeld, Z. Zhu, A. M. C. Dawes, and D. J. Gauthier, "Distortion management in slow-light pulse delay," Opt. Express Vol. 13, pp. 9995-10002, Dec. 2005.

[6] R. W. Boyd, chapter 9 in Nonlinear optics, pp. 409-427. San Diego, CA: Academic Press, 2003.

[7] M. O. vanDeventer, and A. J. Boot, "Polarization properties of stimulated Brillouin scattering in single mode fibers," J. Lightwave Technol. Vol. 12, pp. 585-590, Apr. 1994 